\title{
Assessment of nutritional status of selected military personnel
}

\section{Abstract \\ Introduction}

Problem of overweight and obesity is more and more common in nowadays. Prevalence of overweight and obesity could have longrange consequences in case of organisation where good health and physical efficiency are requested for personnel to perform their professional roles effectively. Many times soldiers need to maintain very high levels of physical activity to perform their duties and to complete demanding missions, which have a huge physical exertion. Because of that soldiers should stay in an excellent health and stamina.

\section{Materials \& methods}

A total of 355 men soldiers from units with diverse specialisation underwent the examination. The most common criterions to estimate abdominal obesity are waist circumference and waist-to-hip ratio. The evaluation was based on determination of body composition measuring fat tissue percentage and antrophometric indexes: weight, height, waist-to-hip ratio (WHR) and waist circumference (WC). Soldiers were classified to abdominal obesity according to WHR (WHR > 1) and WC (WC > 94). The amount of fat tissue was measured using bioelectrical impedance analysis.

\section{Results}

Soldiers were aged 34,56 \pm 7,07 years. Median weight and height were respectively 86,04 $\pm 11,92 \mathrm{~kg}$ and $179,14 \pm 6,02 \mathrm{~cm} .160 \mathrm{soldiers}$ $(45 \%)$ had a waist circumference higher than $94 \mathrm{~cm}$. The waist-to-hip ratio was higher than 1 for $19 \%$ of soldiers. Conducted bioelectrical impedance analysis showed that for $46 \%$ of soldiers percentage of fat tissue exceeded $20 \%$.

\section{Discussion}

The results showed that the problem of overweight and obesity exists also in military populations. In many cases it is connected with incorrect nutritional behaviours. Soldiers require more proactive approach, because the potentially deleterious health outcomes connected with weight gain may have a negative influence on their work. The biggest effort should be put on a proper nutritional education.

\section{Conflict of Interest}

There is no conflict of interest 